\title{
LATTICE-MISMATCHED MONOLITHIC GAAS/INGAAS TWO-JUNCTION SOLAR CELLS BY DIRECT WAFER BONDING
}

\author{
Katsuaki Tanabe ${ }^{1}$, Daniel J. Aiken ${ }^{2}$, Mark W. Wanlass ${ }^{3}$, Anna Fontcuberta i Morral ${ }^{1,4}$ and Harry A. Atwater ${ }^{1,4}$ \\ ${ }^{1}$ Thomas J. Watson Laboratory of Applied Physics, California Institute of Technology, Pasadena, CA 91125 \\ ${ }^{2}$ Emcore Photovoltaics, Albuquerque, NM, 87123 \\ ${ }^{3}$ National Renewable Energy Laboratory, Golden, CO 80401 \\ ${ }^{4}$ Aonex Technologies, Pasadena, CA 91106
}

\begin{abstract}
Direct bonded interconnect between subcells of a lattice-mismatched III-V compound multijunction cell would enable dislocation-free active regions by confining the defect network needed for lattice mismatch accommodation to tunnel junction interfaces, while metamorphic growth inevitably results in less design flexibility and lower material quality than is desirable. The first direct-bond interconnected multijunction solar cell, a two-terminal monolithic GaAs/InGaAs two-junction solar cell, is reported and demonstrates viability of direct wafer bonding for solar cell applications. The tandem cell open-circuit voltage was approximately the sum of the subcell open-circuit voltages. This achievement shows direct bonding enables us to construct lattice-mismatched III-V multijunction solar cells and is extensible to an ultrahigh efficiency InGaP/GaAs/InGaAsP/InGaAs four-junction cell by bonding a GaAs-based lattice-matched InGaP/GaAs subcell and an InP-based lattice-matched InGaAsP/InGaAs subcell. The interfacial resistance experimentally obtained for bonded GaAs/lnP smaller than $0.10 \mathrm{Ohm}-\mathrm{cm}^{2}$ would result in a negligible decrease in overall cell efficiency of $\sim 0.02 \%$, under 1 -sun illumination.
\end{abstract}

\section{INTRODUCTION}

Multijunction solar cells with III-V semiconductor compound materials are promising for ultrahigh (>40\%) efficiency solar energy conversion in the coming decades. Most current multijunction solar cell design approaches are focused on either lattice-matched designs or metamorphic growth with dislocations to accommodate subcell lattice mismatch, which inevitably results in less design flexibility or lower material quality than is desirable. [1,2]

Material integration by the direct wafer bonding technique enable atomic scale semiconductorsemiconductor bonding and do not utilize any metal as bonding agent at interfaces. Thus interface transparency, thermal conductivity, thermal stability and reliability should be superior to mechanical stacking approaches using patterned metallic pastes and frits. Also, monolithic, or two-terminal, devices can be integrated into modules with the same simplicity afforded by single-junction devices, with metallization at the very top and bottom of the stack only. Three- and four-terminal configurations do not require lattice mismatch or current matching. However, they are generally less desirable structures, because of their complexities of fabrication and assembly, than the monolithic device. [3]

Direct wafer bonding enables dislocation-free active regions by confining the defect network needed for lattice mismatch accommodation to heterointerfaces. [4,5] The strain is highly localized to the interface rapidly diminishing away from the interface, implying a minimal disturbance to the bulk of the materials during the wafer bonding process. [6]

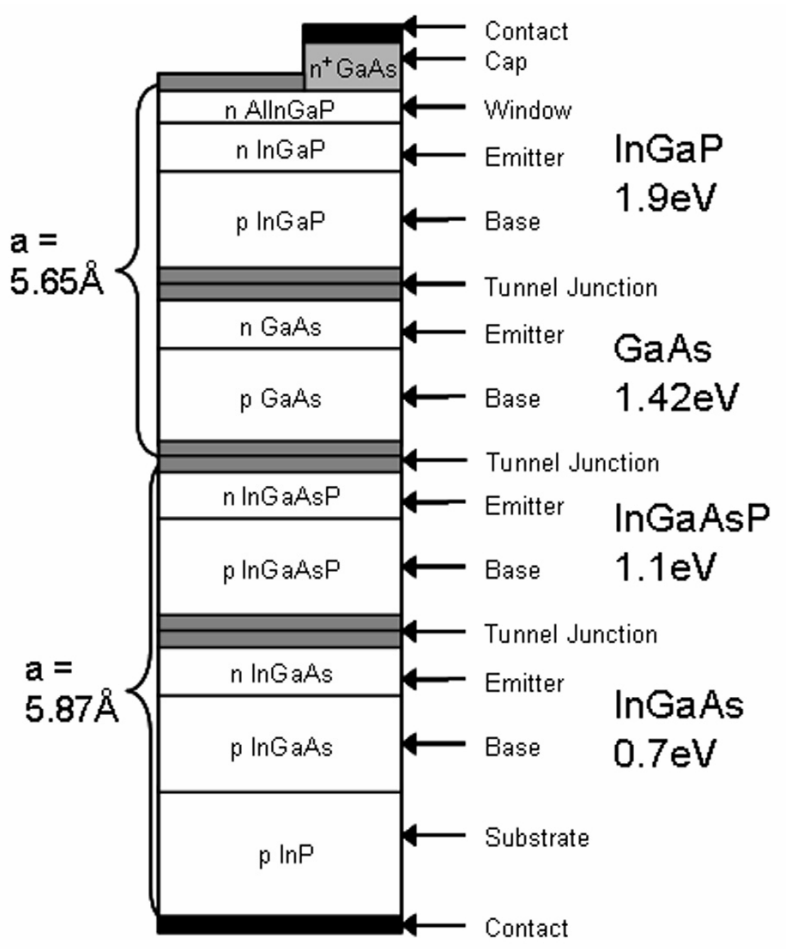

Fig. 1 Schematic cross section of the InGaP/GaAs/InGaAsP/InGaAs four-junction solar cell structure. 
Fig. 1 shows an ideal structure of III-V multijunction solar cell, which could exhibit ultrahigh efficiency. $[7,8]$ It is difficult to prepare this multistack structure by epitaxial growth with optimal material quality because of the $4 \%$ lattice mismatch across the interface of the GaAs and InGaAs subcells. Wanlass et al reported a $37.9 \%$ efficiency cell with an epitaxially grown triple-junction structure of $\mathrm{InGaP} / \mathrm{GaAs} / \mathrm{InGaAs}$. [9] However a considerable density of dislocations limiting the cell efficiency was observed in the InGaAs layer of graded composition. This result suggests the potential of over- $40 \%$ efficiency utilizing the wafer bonding technology to diminish the dislocations.

In the present work, GaAs/InP direct wafer bonding was first investigated for mechanically robust and highly conductive heterointerfaces applicable for solar cell applications. Secondly the first direct-bond interconnected multijunction solar cell, a two-terminal monolithic GaAs/lnGaAs two-junction cell, was fabricated based on the bonding investigation to demonstrate a proof-of-principle for the viability of direct wafer bonding for solar cell applications.

\section{GAAS/INP DIRECT WAFER BONDING}

\section{Experimental}

Direct bonding of heavily doped n-type bulk substrates of (001) GaAs and InP was investigated, with doping concentrations of $2 \times 10^{18} \mathrm{~cm}^{-3} \mathrm{Si}$ doping in GaAs and $4.5 \times 10^{18} \mathrm{~cm}^{-3} \mathrm{~S}$ doping in InP. Interfacial bonding layers prepared by MOCVD growth of thin epitaxial Se-doped GaAs layers on GaAs with and S-doped InP on InP with doping concentration of $1 \times 10^{19} \mathrm{~cm}^{-3}$ (denoted as " $\mathrm{n}^{+}$), were also investigated. The wafers were diced into $\sim 1 \mathrm{~cm}^{2}$ area and bonded following the procedure described elsewhere. [5] Special care was taken to keep the surface of the wafers clean of organic contaminations and particles. After degreasing the surface, the native oxide was removed by dipping the GaAs and InP pieces in 7 vol\%-HCl (aq) and 10 vol\%-HF (aq), respectively, for 30 sec. Then the wafers were brought into contact with the (011) edges aligned. The joined $\mathrm{GaAs} / \mathrm{InP}$ pairs were annealed at $0.5 \mathrm{MPa}$ at $270{ }^{\circ} \mathrm{C}$ in atmosphere for 10 hours followed by annealing in $10 \% \mathrm{H}_{2}$ diluted by $\mathrm{N}_{2}$ at $450-600$ ${ }^{\circ} \mathrm{C}$ for $30 \mathrm{~min}$. Some bonded pairs were subject to only one of these two annealing processes.

The electrical properties of the bonded interfaces were investigated for the different annealing conditions by measuring the current-voltage (I-V) characteristics. An indium solder pad whose area was $\sim 0.05 \mathrm{~cm}^{2}$ was used for Ohmic contact.

\section{Results and discussion}

For multijunction solar cells, formation of mechanically robust, low resistance interfaces is a critical aspect for structure stability and high energy conversion efficiency. Heavy doping at the GaAs and InP interfaces to be subsequently bonded was found to significantly enhance the GaAs/InP interfacial conductivity. I-V characteristics were measured for various combinations of doping concentration to investigate the effect of doping on electrical property. The interfacial I-V curves for the bonded $\mathrm{GaAs} / \mathrm{InP}$ pairs press-annealed at $270{ }^{\circ} \mathrm{C}$ are in Fig. 2. For the forward bias, which is positive on the GaAs side and is the bias direction in the solar-cell operation, the interfacial conductance values were ordered as:

$\mathrm{n}^{+} \mathrm{GaAs} / \mathrm{n}^{+} \mathrm{InP}>\mathrm{n}^{+}$GaAs $/ \mathrm{n} \operatorname{lnP}>\mathrm{n}$ GaAs $/ \mathrm{n} \operatorname{lnP}>\mathrm{n}$ GaAs $/ \mathrm{n}^{+} \operatorname{InP}$.

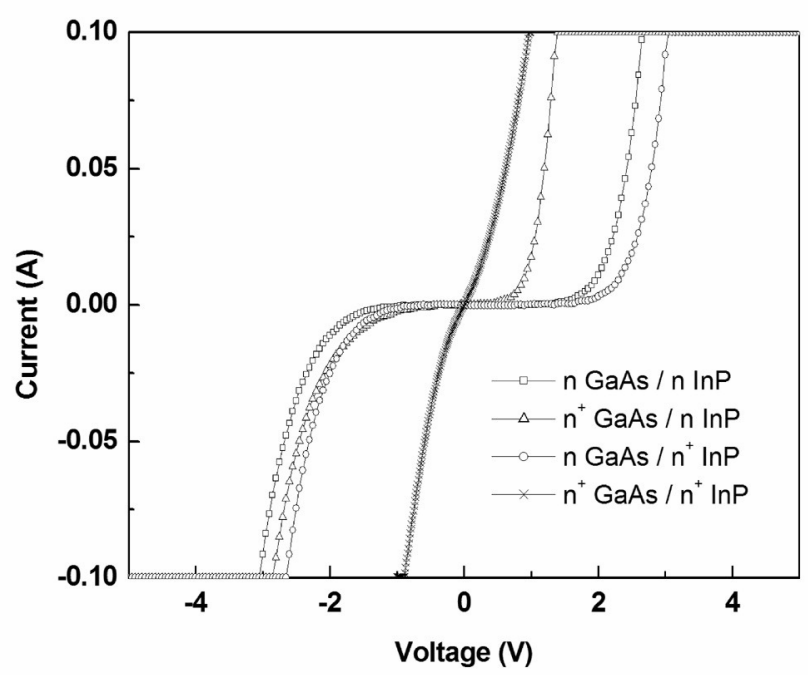

Fig. $2 \mathrm{l}-\mathrm{V}$ characteristics of bonded $\mathrm{GaAs} / \mathrm{lnP}$ heterointerfaces with various doping concentrations bonded at $270{ }^{\circ} \mathrm{C}$.

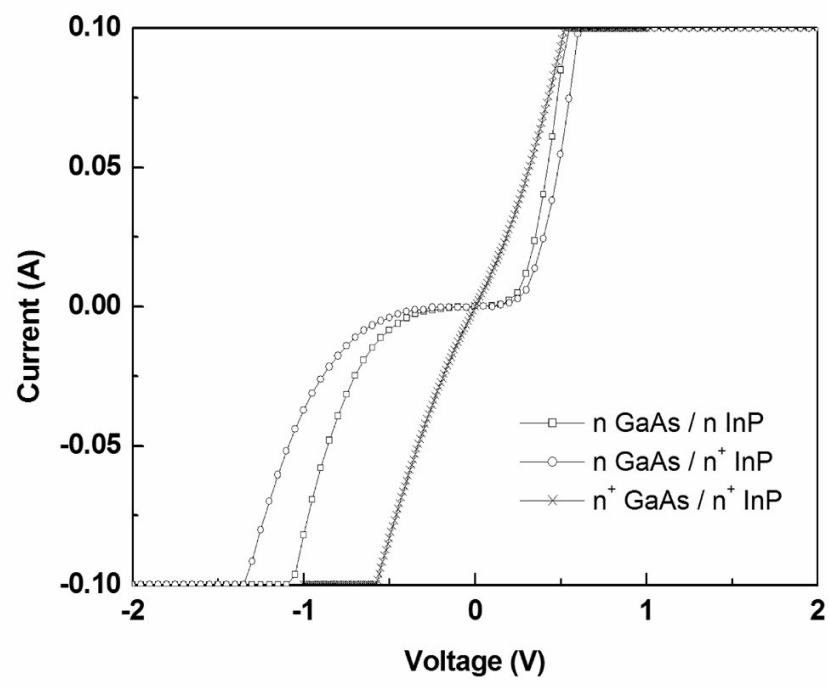

Fig. $3 \mathrm{I}-\mathrm{V}$ characteristics of bonded $\mathrm{GaAs} / \mathrm{lnP}$ heterointerfaces with various doping concentrations bonded at $270{ }^{\circ} \mathrm{C}$ followed by annealing at $550{ }^{\circ} \mathrm{C}$. 


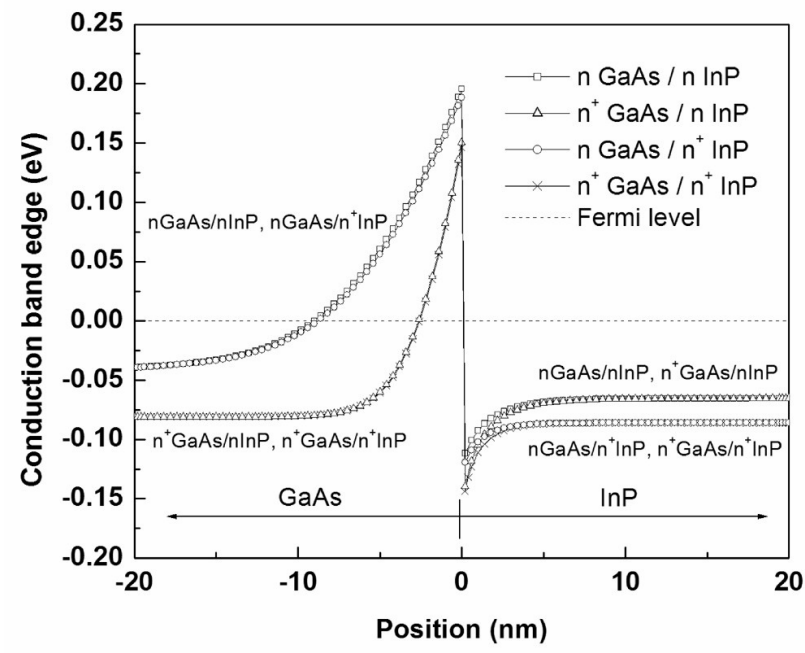

Fig. 4 Calculated profiles of the conduction band edge across $\mathrm{GaAs} / \mathrm{InP}$ heterointerfaces with various doping concentrations.

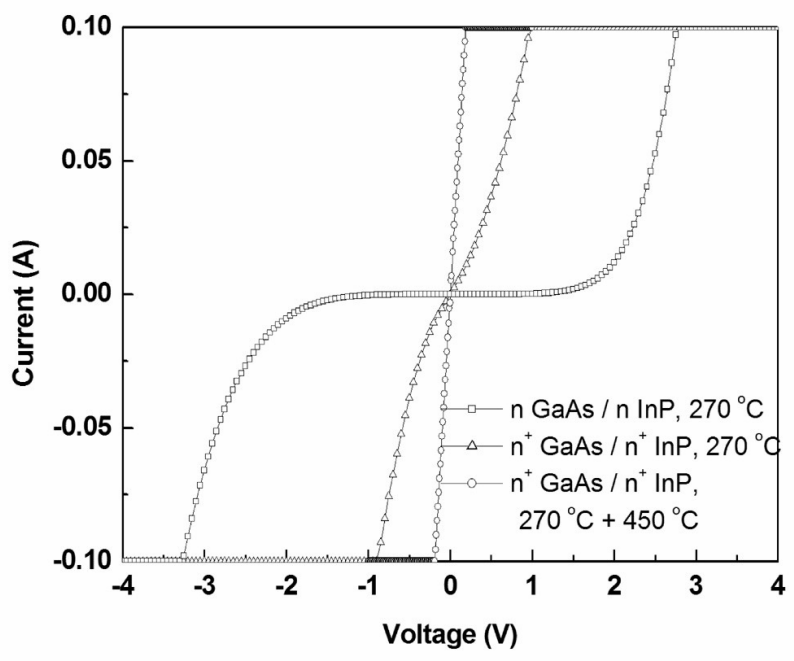

Fig. 5 I-V characteristics of bonded GaAs/InP heterointerfaces with various bonding conditions.

For the reverse bias, which is positive on the $\ln P$ side, the conductance values except of the $\mathrm{n}^{+} \mathrm{GaAs} / \mathrm{n}^{+} \mathrm{InP}$ pair were not clearly different from one another considering the possible error of the measurements. The reason of this similarity however has not been figured out yet. The I-V curves with additional annealing at $550{ }^{\circ} \mathrm{C}$ (Fig. 3) shows the same tendency of Eq. 1, except the $\mathrm{n}^{+} \mathrm{GaAs} / \mathrm{n}$ InP pair, which has not been done.

This tendency of conductivity can be explained by analysis of the heterojunction band offset at the GaAs/InP interface. Electron transport rather than hole transport dominates the current flow in the n-type GaAs and InP used in this study. The conduction-band edge of GaAs is $0.3 \mathrm{eV}$ above that of InP for intrinsic materials. [10] One-dimensional simulations of the heterojunction bandbending indicate a significant decrease in the interface potential barrier width at higher doping concentrations, especially on the $\mathrm{GaAs}$ side of a GaAs/InP heterojunction. [11] (Fig. 4) This barrier thinning enables interfacial tunneling, rather than thermionic emission, leading to higher conductivity across the heterojunction interfaces. [12,13] The $n \ln P$, rather than $n^{+} \ln P$, has the higher conduction-band edge by $0.02 \mathrm{eV}$ referenced to the homogeneous bulk, which aids interface transport. However, sulfur diffusion from $\mathrm{InP}$ to $\mathrm{GaAs}$, which was observed by SIMS measurement, can cause barrier thinning. These factors may determine the $\mathrm{I}-\mathrm{V}$ characteristics all in all.

Ideally, Ohmic GaAs/InP heterojunctions would be formed by bonding at lower temperature to avoid possible degradation of the cell interfaces and $p-n$ junctions for multijunction solar cell applications. The approach taken here yielded Ohmic interfaces with $<0.10 \mathrm{Ohm}-\mathrm{cm}^{2}$ interface resistance at as low as $450{ }^{\circ} \mathrm{C}$ and $<1.0$ $\mathrm{Ohm}-\mathrm{cm}^{2}$ by solely pressure annealing at $270{ }^{\circ} \mathrm{C}$ in $\mathrm{n}^{+} \mathrm{GaAs} / \mathrm{n}^{+} \mathrm{InP}$ structures, as shown in Fig. 5 .

\section{GAAS/INGAAS TWO-JUNCTION SOLAR CELLS}

\section{Experimental}

In the second phase of this study, a two-terminal monolithic, two-junction tandem solar cell was fabricated from direct bonding of single-junction $\mathrm{GaAs}$ and InGaAs subcells. The GaAs subcell consisted of $p$ and $n$ type layers of GaAs epitaxially grown on a (001) GaAs substrate by MOCVD. The InGaAs subcell had a bandgap energy of $0.74 \mathrm{eV}$ and consisted of $p$ and $n$ type layers of InGaAs

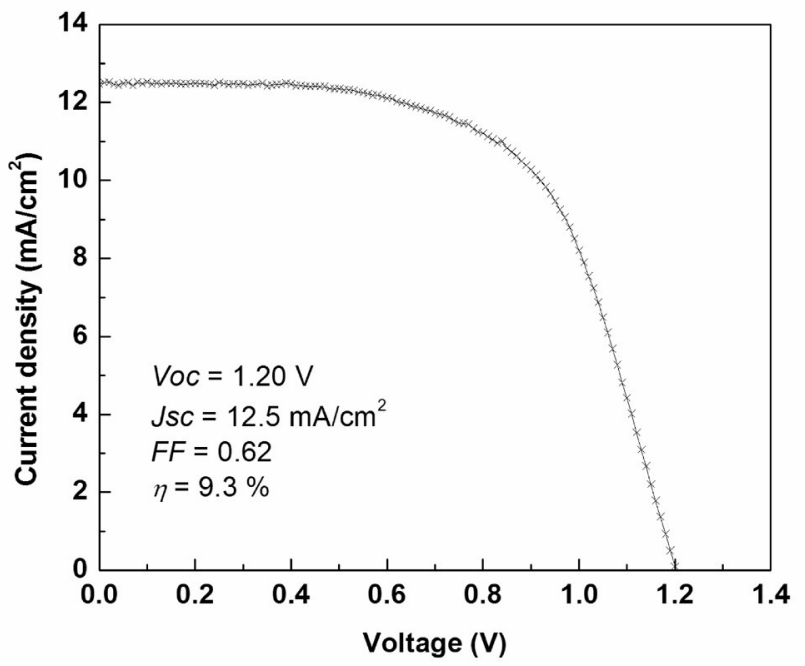

Fig. $6 \mathrm{I}-\mathrm{V}$ curve for the bonded $\mathrm{GaAs} / \mathrm{InGaAs}$ solar cell at 1 sun, AM1.5G. 
layers lattice-matched to (001) InP. Specifically, the GaAs subcell was terminated with a Se-doped GaAs layer with $1 \times 10^{19} \mathrm{~cm}^{-3}$ carrier concentration and the InGaAs subcell was terminated with a S-doped $\operatorname{InP}$ layer with carrier concentration of $2 \times 10^{19} \mathrm{~cm}^{-3}$. After bonding of the two subcells, the GaAs substrate was removed to complete a $\mathrm{GaAs} / \mathrm{InGaAs} / \mathrm{InP}$ heterostructure forming the two-junction solar cell. These subcells were bonded as described above and annealed at $0.5 \mathrm{MPa}$ at $380{ }^{\circ} \mathrm{C}$ for 10 hours followed by annealing in $\mathrm{H}_{2} / \mathrm{N}_{2}$ at $350{ }^{\circ} \mathrm{C}$ for 30 min after metallization with Au. Photovoltaic I-V characteristics of the bonded $\mathrm{GaAs} / \mathrm{InGaAs}$ two-junction cell were measured with $0.337 \mathrm{~cm}^{2}$ active illumination area under AM1.5 Global solar spectrum with 1-sun total intensity $\left(100 \mathrm{~mW} \mathrm{~cm}^{-2}\right)$. For comparison, photovoltaic I-V characteristics of the unbonded $\mathrm{GaAs}$ and InGaAs subcells were also measured. Each of the subcells was processed in the same way as the bonded $\mathrm{GaAs} / \mathrm{InGaAs}$ cell. The GaAs subcell was mounted on a handling glass substrate with gold film via conductive silver-epoxy glue, for its inversely-grown structure, and the original GaAs substrate was removed by chemical etching.

\section{Results and discussion}

The photovoltaic I-V characteristics of the bonded GaAs/InGaAs two-junction solar cell are shown in Fig. 6 . The device parameters for this cell were $J_{s c}=12.5 \mathrm{~mA} \mathrm{~cm}^{-2}$, $V_{o c}=1.20 \mathrm{~V}, F F=0.62$, and $E f f=9.3 \%$, where $J_{s c}, V_{o c}, F F$ and Eff are short-circuit current, open-circuit voltage, fill factor and energy conversion efficiency, respectively. The low fill factor may be accounted for by series resistance in the contacts, which can be lowered by contact redesign. Surface passivation and optimization of cell assembly parameters, such as current matching, would also give further improvement of the cell efficiency. The $V_{o c} s$ of the unbonded $\mathrm{GaAs}$ and InGaAs subcells were $0.91 \mathrm{~V}$ and $0.27 \mathrm{~V}$. Thus, the $V_{o c}$ of the bonded GaAs/InGaAs two-junction cell was approximately equal to the sum of the open circuit voltages for the GaAs and InGaAs subcells. This $V_{o c}$ result indicates that the bonding process does not degrade the cell material quality since any generated crystal defects that act as recombination centers would reduce $V_{o c} \cdot[14,15]$

\section{CONCLUSIONS}

In this paper we demonstrated use of direct wafer bonding in a tandem solar cell. Such an approach can also be applied to other photovoltaic heterojunctions where lattice mismatch accommodation is also a challenge, such as the InGaP/GaAs/InGaAsP/InGaAs four-junction tandem cell by bonding a GaAs-based lattice-matched InGaP/GaAs subcell to an InP-based lattice-matched InGaAsP/InGaAs subcell. Simple considerations suggest that for such a cell the currently-reported interfacial resistance smaller than $0.10 \mathrm{Ohm}-\mathrm{cm}^{2}$ would result in a negligible decrease in overall cell efficiency of $\sim 0.02 \%$, under 1-sun illumination. [8]

\section{ACKNOWLEDGEMENTS}

This work was supported by NASA and the National Renewable Energy Laboratory. Alireza Ghaffari, Robert Walters of the California Institute of Technology and James Zahler of the Aonex Technologies are acknowledged for their technical support in development of the bonding process and the electrical measurements. One of the authors (KT) was supported in part by the Japanese ITO Scholarship for International Education Exchange.

\section{REFERENCES}

[1] S. Mahajan, Acta Mater. 48, 2000, pp. 137-149.

[2] Y. Shimizu and Y. Okada, J. Cryst. Growth 265, 2004, pp. 99-106.

[3] J. M. Olson, D. J. Friedman, and S. R. Kurtz, in Handbook of Photovoltaic Science and Engineering, edited by A. Luque and S. Hegedus (Wiley, New York), 2003, pp. 359-411.

[4] U. Goesele and Q. Y. Tong, Ann. Rev. Mater. Sci. 28, 1998, pp. 215-241.

[5] A. Fontcuberta i Morral et al, Appl. Phys. Lett. 83, 2003, pp. 5413-5415.

[6] Z. L. Liau, Phys. Rev. B 55, 1997, pp. 12899-12901.

[7] J. M. Zahler et al, Proc. 29th IEEE Photovoltaic Specialists Conference, 2002, pp. 45-48.

[8] P. R. Sharps et al, Proc. 26th IEEE Photovoltaic Specialists Conference, 1997, pp. 895-898.

[9] M. W. Wanlass et al, MWW 3rd ISCC, 2005.

[10] S. Tiwari and D. Frank, Appl. Phys. Lett. 60, 1992, pp. 630-632.

[11] PC1D Version 5.2 (University of New South Wales), 1998.

[12] A. Y. C. Yu, Solid-St. Electron. 13, 1970, pp. 239-247.

[13] H. Sasaki et al, Appl. Phys. Lett. 31, 1977, pp. 211-213.

[14] R. B. Bergmann, Appl. Phys. A 69, 1999, pp. 187-194.

[15] R. Brendel, Jpn. J. Appl. Phys. 40, 2001, pp. 4431-4439. 